\title{
Chromatic Dispersion of an Optical Fiber Based on Photonic Quasicrystals with Twelve- Fold Symmetry and its Application as Directional Coupling
}

\author{
Ádller de O. Guimarães ${ }^{1,3}$, José P. da Silva ${ }^{1,2}$ and Emmanuel R. M. Dantas ${ }^{1}$ \\ ${ }^{1}$ Federal University of Rio Grande do Norte, Av. Senador Salgado Filho, 3000, 59078-970, Natal, RN, Brazil \\ ${ }^{2}$ Federal University of Semiarid Region, Av. Francisco Mota, 572, 59625-900, Mossoró, RN, Brazil \\ ${ }^{3}$ Federal University of Semiarid Region, BR 226, KM 405, 59900-000, Pau dos Ferros, RN, Brazil \\ adller.guimaraes@ufersa.edu.br,patroc@ct.ufrn.brand manel.mrq@gmail.com
}

\begin{abstract}
Optical fibers composed by photonic quasicrystals it is based on aperiodic structures characterized by at least two different symmetrical patterns from the base matrix. In this work, an optical fiber composed by quasi-periodic and symmetric matrix was analyzed using the finite element method in conjunction with cylindrical perfectly matched layers. The structure is composed by pure silica and/or silica doped with germanium, and originated from twelve distributions of air holes symmetrically with a defect caused by the absence of the fiber central hole. The obtained results shown that the structure in analysis exhibits an ultra-flat chromatic dispersion for a range of wavelengths varying from 1.4 to $1.6 \mu \mathrm{m}$ covering the bands $E, S, C$ and $L$, with chromatic dispersion, for the silica doped with germanium, varying between 22.1 and 23.01 $\left[p s . \mathrm{km}^{-1} \cdot \mathrm{nm}^{-1}\right]$. This photonic quasicrystal fiber (PQCF) was used to form a coupler with three identical horizontal cores surrounded by air holes. The signal power launched in the central core is equally divided between the two neighboring cores with $50 \%$ of the coupling ratio.
\end{abstract}

Index Terms - Photonic Quasicrystals Fibers, Finite Elements and 12-fold symmetry.

\section{INTRODUCTION}

Structures composed by Photonics Quasicrystal (PQC) represent a class of structures that has been extensively studied in the last decade, mainly due to their application in the development of optical fibers [1]. These types of structures exhibit an important feature, do not contain translational symmetry, normally present in traditional photonic crystals (PCs).

A photonic crystal is a structured material such that its dielectric function varies periodically in space and can be considered, depending on the application, as a periodic optical nanostructures designed to affect the motion of photons in a similar manner to the periodicity of a semiconductor crystal would affect the motion of electrons [2]. Moreover, the quasicrystals are characterized by a very unusual alignment of the atoms and containing aperiodic structures, they have at least two different symmetrical patterns from the base matrix, which also form a structure without gaps, but not regularly repeating [3]. Thus, under these circumstances, it can lead symmetries of $6,8,12$ and even 
24- fold. The PQCFs have the same principle of manufacturing of PCFs, i.e. they are usually manufactured using the pure silica, doped silica or polymers as the base material and the microstructure of the shell is obtained with inclusions of smaller refractive index, usually filled air holes or some material with smaller refractive index than the base material [2]. The most complex design of a PQCF requires refined techniques of manufacturing and process control. The main geometrical parameters of a solid core PQCF with holes distributed from a quasi-periodic matrix with 12-fold symmetry are the number of repetitions of the basic matrix consisting of air holes, the diameter of holes and the distance between consecutive holes. In addition, the size of the holes can be adjusted from the temperature control. [4], [5]. However, Photonics Quasicrystal Fibers (PQCF) has attracted the attention of the scientific community, due to its ability to present symmetry in higher orders, as for example symmetry with twelve replicates (twelve-fold). This important feature allows the development of optical fibers with a flat chromatic dispersion for several wavelengths.

In addition, with the advent of microstructured fibers composed by photonic quasicrystals, in which air holes are distributed in a matrix of silica or doped silica over the entire length of the fiber, it has become possible to obtain multiple arrangements of air holes arranged symmetrically in a single fiber [6]. This feature puts PQCFs as versatile structures for obtaining flat chromatic dispersion. For dispersion compensation in a wide wavelength range, parameters as variation of dispersion and maximum slope must be simultaneously compensated. The compensation is obtained with the use of optical compensating fibers specially designed for this purpose. In addition for use of optical fibers, including the PQCFs, as residual dispersion compensator, these should have a flat dispersion and opposite sign to the residue. Thus, it is possible to compensate for a wavelength range and prevent the accumulated dispersion in long connections that limit the operation of the optical link. [7].

Due to its ability to produce light in hollow cores, photonic quasi crystal fiber can be used in various applications of optical communications, one of them is their application as optical coupler. In addition the multicore optical fibers play a significant role in dividing/combining the power in optical fiber networks [8]-[10]. On these fiber models, a single power may be divided through the cores in the structure and forwarded to different areas for additional purposes. In this context, several approaches have been adopted to analyze the division process of optical powers among the $N$ cores in the structure. The most common approach is the fusion of several identical or nonidentical optical fibers together by keeping mutual contact in the fusion region [11].

On the other hand, with the photonic crystal and quasi crystal fiber advent, also called microstructured fibers (MF), in which air holes are distributed on a silica matrix throughout the fiber length, it became possible to obtain multiple cores in a single fiber, eliminating the fusion process [11]. This characteristic puts the PQCFs as versatile structures in obtaining power divisors devices.

In this paper, the finite element method in conjunction with perfectly matched layers (PML) of cylindrical type [11] are used in the analysis of a new model of optical fiber based in photonic quasicrystals with twelve-fold symmetry. The proposed structure is characterized by employing of an 
aperiodic matrix of microscopic air holes, forming a symmetrical arrangement with twelve replications Fig. 1. The air holes are separated by a distance $\Lambda$ and have equal diameters $(d)$. In addition the PQCF is used to form a coupler with three identical horizontal cores surrounded by air holes; in this case, the structure consists of two identical cores horizontally equidistant from the central core which is also identical. The cores are distributed to allow the maximum energy transfer among them. In the next section, the finite element formulation including PML of cylindrical type, concepts about project of photonic quasicrystals fibers are described in section III, numerical results are shown in section IV, and finally are presented the conclusions of this work.

\section{METHOD OF ANALYSIS}

The finite element method is widely recognized as a powerful tool for numerical analysis of optical devices [12]-[13]. The approximation by vector finite elements for analysis of propagation characteristics in optical fibers with high precision has been studied and applied successfully in many different structures. In this regard, in communications systems involving optical fibers, knowledge of the chromatic dispersion $D$ becomes extremely important to determine the quality of the pulse's transmission, because this factor is directly responsible for the width pulse. Moreover, $D$ is proportional to the second derivative of the propagation constant or effective refractive index $n_{\text {eff }}$. Thus, a highly accurate numerical tool as the finite element method [11], it becomes necessary to obtain the $n_{\text {eff. }}$.

In addition, besides the application of the numerical method based on finite element, the Sellmeier coefficients are used in the formulation to examine the chromatic dispersion, the variation of effective index and effective area of the fundamental mode in different frequencies. Sellmeier coefficients are applied directly in the vector formulation, in which synthesis is obtained starting from the Helmholtz vector equation in two dimensions and considering perfectly matched layers (PMLs) to avoid unwanted reflections. Thus, one can write:

$$
\nabla \times(\bar{k} \nabla \times \vec{H})-k_{0}^{2} \vec{H}=0
$$

where $\overline{\bar{k}}=1 /(\bar{\varepsilon} \overline{\bar{L}})$, and $\bar{\varepsilon}$ represents the relative permissiveness tensor, and $\overline{\bar{L}}$ is a tensor relating to parameters of the cylindrical PML [12]. After some algebraic manipulations and assuming that both the means and the fields vary slowly along the propagation direction, $z$ [12], [13], the equation (1) can be rewritten as:

$$
[A]\{\phi\}=n_{e f f}^{2}[B]\{\phi\}
$$

where $[A]$ and $[B]$ are sparse and complex matrices. Equation (2) is efficiently solved through the method on the interaction of subspaces. It is worth pointing that the refractive index profile $n(r, \lambda)$ of an optical fiber can be written as $n(r, \lambda)=\eta(r) n_{s}(\lambda)$, where $n_{s}(\lambda)$ is the refractive index of pure silica and $\eta(r)$ is the normalized refractive index, which is a function only of the radial coordinate.

In this case, the Sellmeier constants for the refractive index of silica $n_{s}(\lambda)$ given in [9] are 
considered in terms of its percentage of doping. Thus, the material dispersion is included directly in the calculations. The use of Sellmeier coefficients in this type of numerical analysis is justified because they can adjust to experimental measurements of refractive index of pure silica and its variations which include percentages of doping. Here, $n_{\text {eff }}$ is obtained from equation (2) considering a band of wavelengths and chromatic dispersion can be directly calculated from the following expression $D=-(\lambda / c)\left(d^{2} n_{\text {eff }} / d \lambda^{2}\right)$, where $c$ is the speed of light in free space and $\lambda$ the wavelength.

In addition we use the vectorial finite element method (FEM) in conjunction with beam propagation method to formulate the Vectorial Beam Propagation Method (VBPM) [7] for solving Maxwell's equations that govern the propagation analysis of field distribution in the cross section of coupler obtained from photonic quasycristal fiber with three cores.

\section{PQFC DESIGN}

The Fig. 1 shows the elementary unit of a photonic quasicrystal and the cross section of PQCF with twelve-fold symmetry, proposed in this paper. For analysis of the dispersion, the central region of the cores 2 and 3 are put air holes and the central region, i.e. in the fiber core 1, the air hole is substituted by pure silica doped with germanium. For analysis of the chromatic dispersion, in all numerical calculations the refractive index of the material that constitutes the region without air holes is of silica doped with germanium, obtained through the Sellmeier equations [14]. Preliminary studies show that the dispersion for the structure in question is flat over bands $E+S+C+L$. Numerical calculations were performed to obtain the fundamental mode of PQCF was for a wavelength of $1.55 \mu \mathrm{m}$.

Next, we analyzed the coupler obtained from a PQCF with three cores as showed in Fig. 1. In this case the refractive index of the material that constitutes the region without air holes is made of pure silica and the air holes with numbers 2 and 3 are substituted by pure silica forming one coupler with three cores numbered as shown in Fig. 1. 


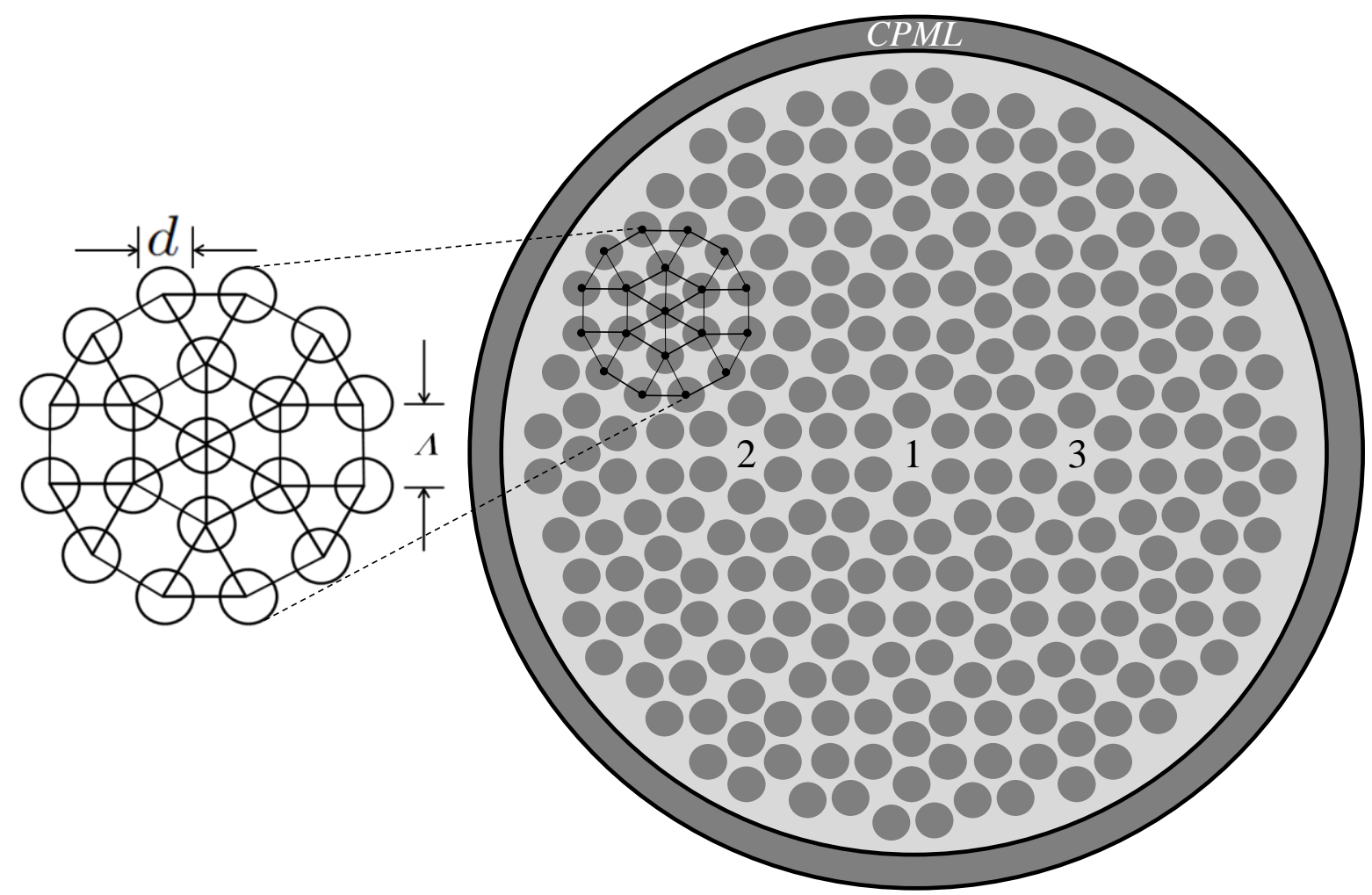

Fig. 1. Left side structure of a photonic quasicrystal and right side cross section of photonic quasicrystal fiber with twelvefold symmetry and cores numbered from 1-3.

Structures quasi-periodic of the type quasicrystals are unique structures that have long range in the direction of propagation, but without periodicity. Recently, studies have found that quasi-periodic structures can originate an important phenomena and properties which are not observed in periodic structures [3], [4]. For example, introduction of 12-fold symmetry in quasicrystals through air holes in a dielectric matrix with low refractive index creates photonic gaps, i.e., frequency bands in which the light propagation is totally prohibited, except for the case of some PCFs settings that allow the propagation of light through the Photonic Band Gap (PBG) [4],[15].

In this work, the behavior of the chromatic dispersion was presented as a function of structural parameters for the fiber shown in Fig. 1. Initially, to obtain the chromatic dispersion, the value of $d / \Lambda$ is fixed in 0.31 and the diameter of the air holes are varied as: $d=0.70 \mu \mathrm{m}, d=0.75 \mu \mathrm{m}, d=0.77 \mu \mathrm{m}$ e $d=0.80 \mu \mathrm{m}$. Then, the value of $d$ was held constant in $0.75 \mu \mathrm{m}$, while the value of the ratio $d / \Lambda$ ranged of \pm 0.10 , i.e., $d / \Lambda=0.31 \pm 0.10$. Is worth mentioning that a reduction in the ratio $d / \Lambda$, with constant $d$, implies an increase of $\Lambda$, representing an growth in fiber thickness and a increment in the ratio $d / \Lambda$, with constant $d$, implies a decrease of $\Lambda$, representing a decrement thickness of the fiber. Just as the photonic crystal fibers, the PQCF can support a single propagation mode, this causes some geometries considered monomode [5].

To obtain the fundamental mode of the fiber, the formulation briefly presented in previous section, was used to calculate the eigenvalues present in the structure of PQCF with twelve-fold symmetry, 
considering $d=0.75$ e $d / \Lambda=0.31$, the computational domain was divided for approximately 30,000 triangular elements, considering external radius for PQCF of $21 \mu \mathrm{m}$. For the calculations considering $d / \Lambda=0.21$ and $d / \Lambda=0.41$, the PQCF was divided in 25,000 and 35,000 linear elements, respectively.

\section{RESULTS}

The simulations showed that in a Photonic Crystal Fiber (PCF) the air holes cause a decrease in the average refractive index of the cladding region of the fiber and confines light in the solid. Thus, the light propagation in PCF occurs due to total internal reflection which describes the light guiding in the central core of solid [3], this characteristics so occurs in the PQCF with 12-fold symmetry. This type of fibers becomes despicable losses in relation to the propagated signal range. Here, the Sellmeier equations for silica doped with germanium were used to obtain the refraction index as a function of wavelength.

Preliminary results showed that the use of meshes with a very larger number of elements than those experienced in this analysis does not improve the accuracy of numerical results. Fig. 2 shows the chromatic dispersion for PQCF with twelve-fold symmetry as a function of wavelength for a band ranging from $1.25 \mu \mathrm{m}$ to $2.0 \mu \mathrm{m}$. Fig. 3 shows the chromatic dispersion for the fundamental mode of PQCF considering the variation of the wavelength within the optical frequency spectrum, i. e., wavelength varying from $1.4 \mu \mathrm{m}$ to $1.6 \mu \mathrm{m}$. It may be noted that small variations in the diameter of the air holes, while maintaining fix the relation $d / \Lambda$, represent important changes in the value of chromatic dispersion. For the PQCF with $d=0.75$ and $d / \Lambda=0.31$, the dispersion varies between 22.1 and $23.01\left[\mathrm{ps} . \mathrm{km}^{-1} \cdot \mathrm{nm}^{-1}\right]$, for a range of wavelength varying from 1.4 to $1.6 \mu \mathrm{m}$. For this range of wavelength the chromatic dispersion is ultra-flat, which suggests their application as chromatic dispersion compensation device.

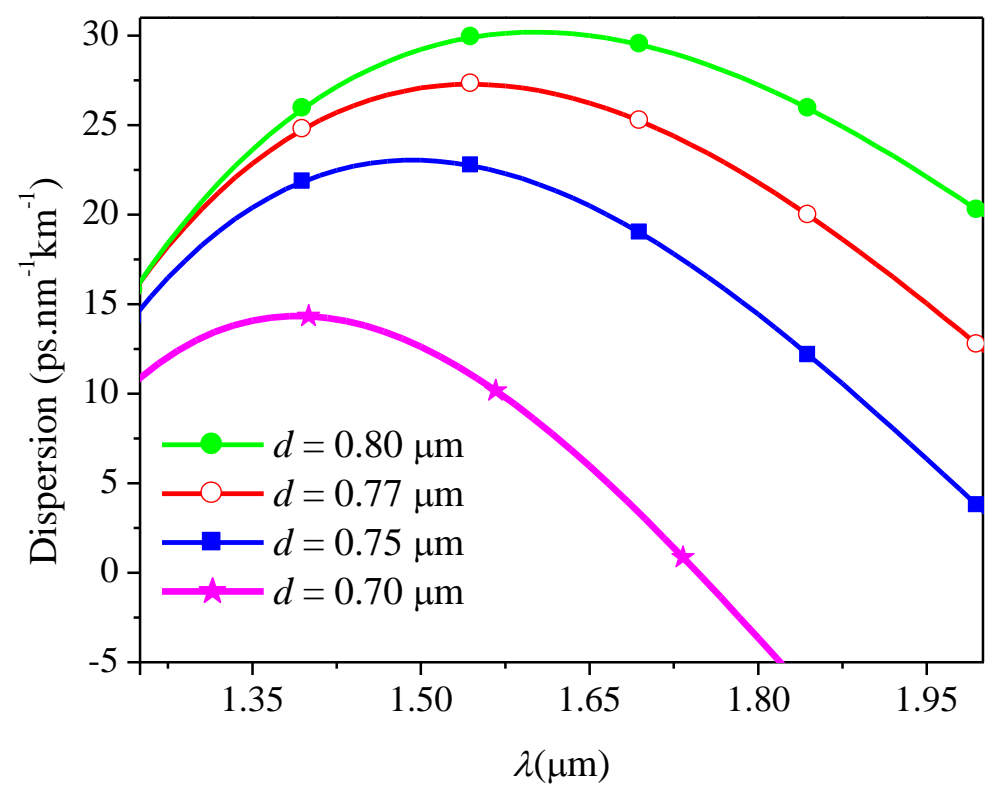

Fig. 2. Chromatic dispersion for the fundamental mode of the structure with twelve-fold symmetry considering $d / \Lambda=0.31$ for a range of wavelength varying from $1.25 \mu \mathrm{m}$ to $2.0 \mu \mathrm{m}$. 
The Fig. 4 shows the comparison between the chromatic dispersion of the fundamental mode for PQCF with $d=0.75$ e $d / \Lambda=0.31$ considering the structure with symmetry 6 and 12 -fold and the variation of the wavelength within the optical frequency spectrum, i. e., wavelength varying from $1.4 \mu \mathrm{m}$ to $1.6 \mu \mathrm{m}$.

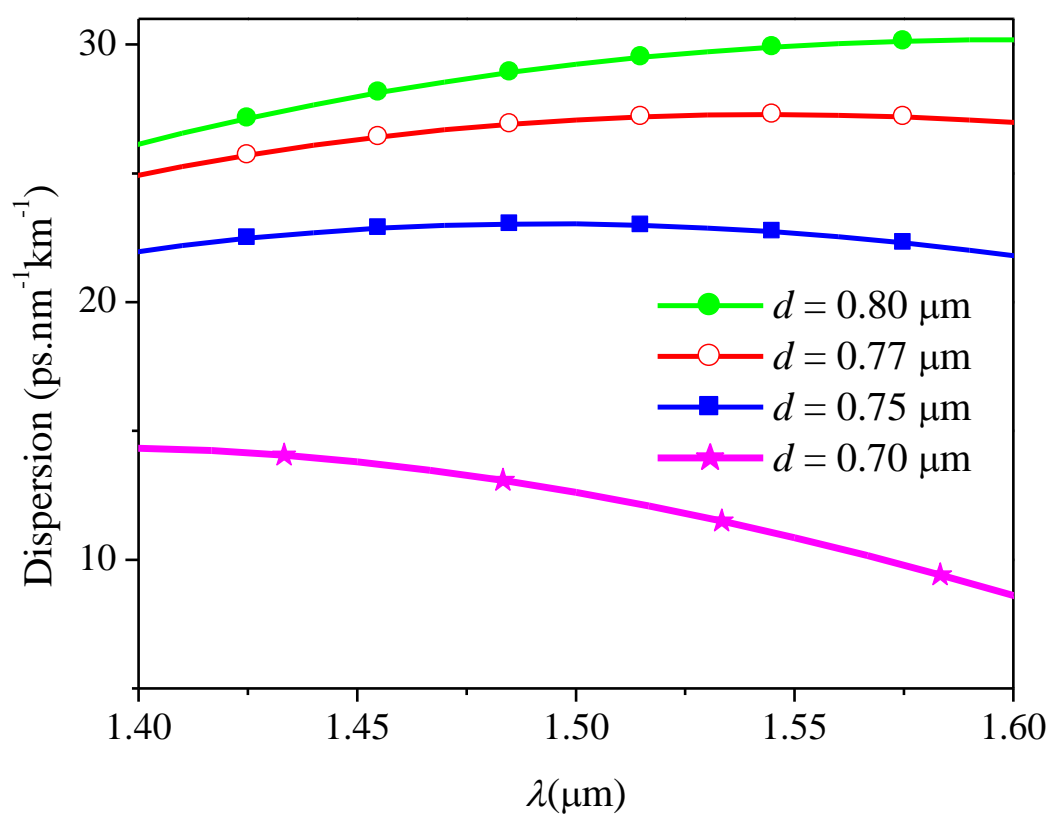

Fig. 3. Chromatic dispersion for the fundamental mode of the structure with twelve-fold symmetry considering $d / \Lambda=0.31$ for a range of wavelength varying from de 1.4 to $1.6 \mu \mathrm{m}$.

In addition, when considering only the range of wavelengths of the optical spectrum, i.e., $\lambda$ vary from 1.4 to $1.6 \mu \mathrm{m}$, the chromatic dispersion of PQCF, for the two configurations, can be considered ultra-flat with values of dispersion within the corresponding optical spectrum bands E, S, C and L. The structure with 12-fold symmetry has dispersion slightly smaller than the structure with 6-fold symmetry [16].

Considering the structure with 12-fold symmetry, the chromatic dispersion slope was 0.0091 [ps.km ${ }^{-1} . \mathrm{nm}^{-2}$ ] for the $E$ band (1.360-1.460 nm) and 0.00015 ps. $\mathrm{km}^{-1} . \mathrm{nm}^{-2}$ for the $L$ band (1.565-1.625 $\mathrm{nm}$ ), with values of $22.555 \pm 0.455\left[\mathrm{ps} . \mathrm{km}^{-1} \cdot \mathrm{nm}^{-1}\right]$. For purposes of design, in [17], the authors consider as flat a dispersion with variation of approximately $17\left[\mathrm{ps} . \mathrm{km}^{-1} . \mathrm{nm}^{-1}\right]$, for a bandwidth of $0.35 \mu \mathrm{m}$. In this work, the variation of dispersion $(\Delta D)$ was approximately $0.91\left[\mathrm{ps} \cdot \mathrm{km}^{-1} \cdot \mathrm{nm}^{-1}\right]$, for a bandwidth of $0.2 \mu \mathrm{m}$. If the bandwidth is adjusted to operate between $1.44 \mu \mathrm{m}$ and $1.46 \mu \mathrm{m}$ corresponding only the optical bands $S, C$ and $L$, as suggested in [18], the variation of dispersion obtained for the PQCF proposed is $0.35\left[\mathrm{ps} . \mathrm{km}^{-1} \cdot \mathrm{nm}^{-1}\right]$ and its maximum slop $-0.027\left[\mathrm{ps} . \mathrm{km}^{-1} \cdot \mathrm{nm}^{-2}\right]$, which shows that the model proposed has a ultra-flat dispersion. 


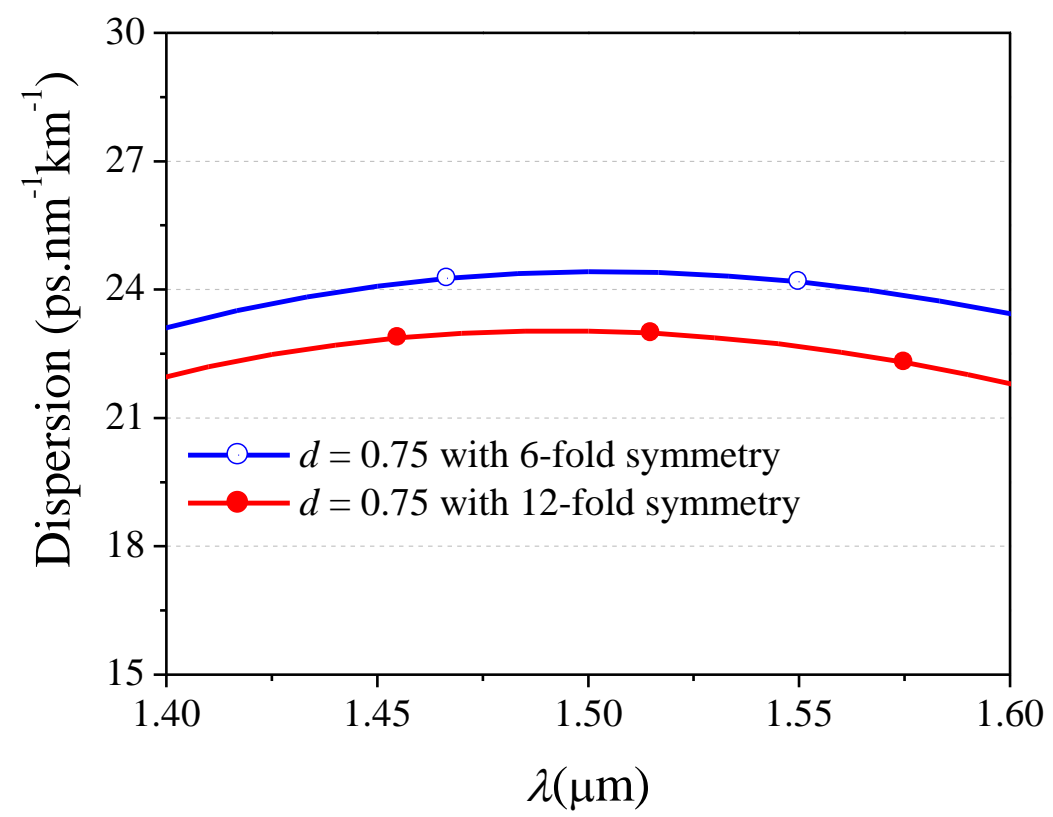

Fig. 4. Comparison between chromatic dispersions for the fundamental mode of PQCF with 12 and 6-fold symmetries, considering $d / \Lambda=0.31$ and $d=0.75 \mu \mathrm{m}$.

The fiber proposed in this work presents characteristics of a single mode fiber, as can be seen in Fig. 5. The fundamental mode corresponds to a mode $H_{X}^{11}$ and to reinforce this tendency, Fig. 5 shows the chromatic dispersion obtained to the first and second propagating modes, the fundamental mode and the second mode, considering the effective refractive index $\left(n_{\text {eff }}\right)$ calculated by the ratio $\beta / k_{0}$, it can be seen that the modes are not degenerate. However, preliminary results show that the presence of air holes may influence the cut-off frequency of the propagating modes in relation to the solid fiber core. Thus, small variations in the diameter of the air holes and/or in the ratio $d / \Lambda$, may influence the configuration of the modal characteristics of the PQCF.

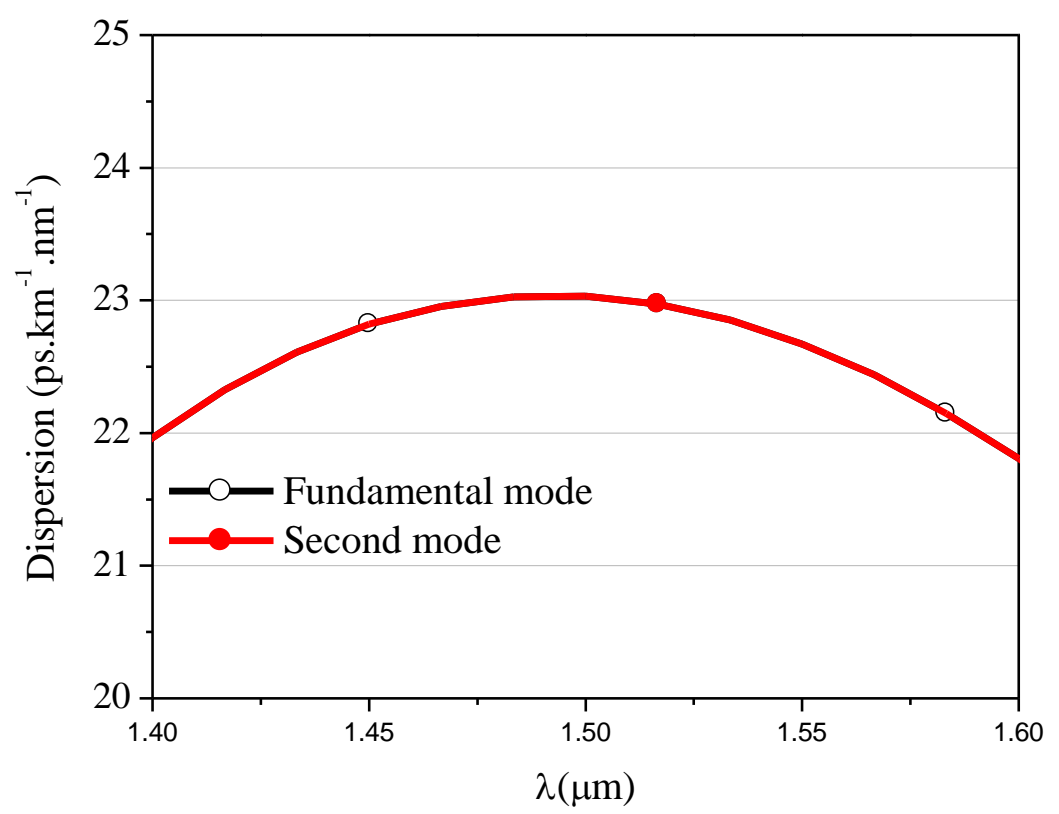

Fig. 5. Chromatic dispersion for the first and second PQCF modes for 12 -fold symmetry considering $d / \Lambda=0.31$ and $d=0.75 \mu \mathrm{m}$. 

http://dx.doi.org/10.1590/2179-10742015v14i2481

The Fig. 6 shows the variation of the effective area for the fundamental mode in function of wavelength for the PQCF with 6 and 12-fold symmetries. The effective area $\left(A_{\text {eff }}\right)$ is a quantitative measure of the area actually covered by the fiber mode in relation to the transverse dimensions. The effective area was calculated using the following expression [19]:

$$
A_{e f f}=\frac{\left(\iint|\vec{E}|^{2} d x d y\right)^{2}}{\iint|\vec{E}|^{4} d x d y}
$$

where $\vec{E}$ represents the electric field.

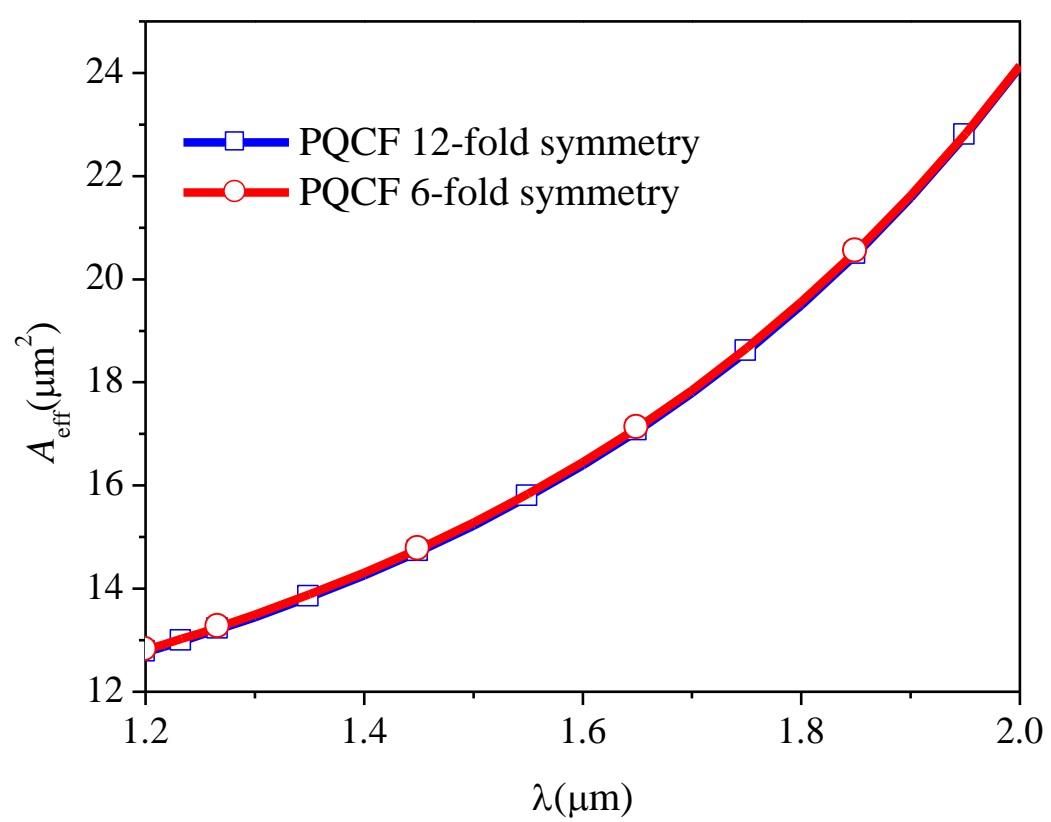

Fig. 6. Effective area of the fundamental mode for the PQCF with 12-fold symmetry and 6-fold symmetry considering $d=$ 0.75 and $d / \Lambda=0.31$.

As can be seen in Fig. 6, the effective area for the two configurations of the fibers in analysis are equivalent, this is due to the regions of confinement of the electric field be equal for both configurations.

The Fig. 7 shows the chromatic dispersion for the fundamental mode of the structure with 12-fold symmetry considering variations of $d / \Lambda$ in \pm 0.10 for a range of wavelengths varying from 1.25 to $1.8 \mu \mathrm{m}$. 


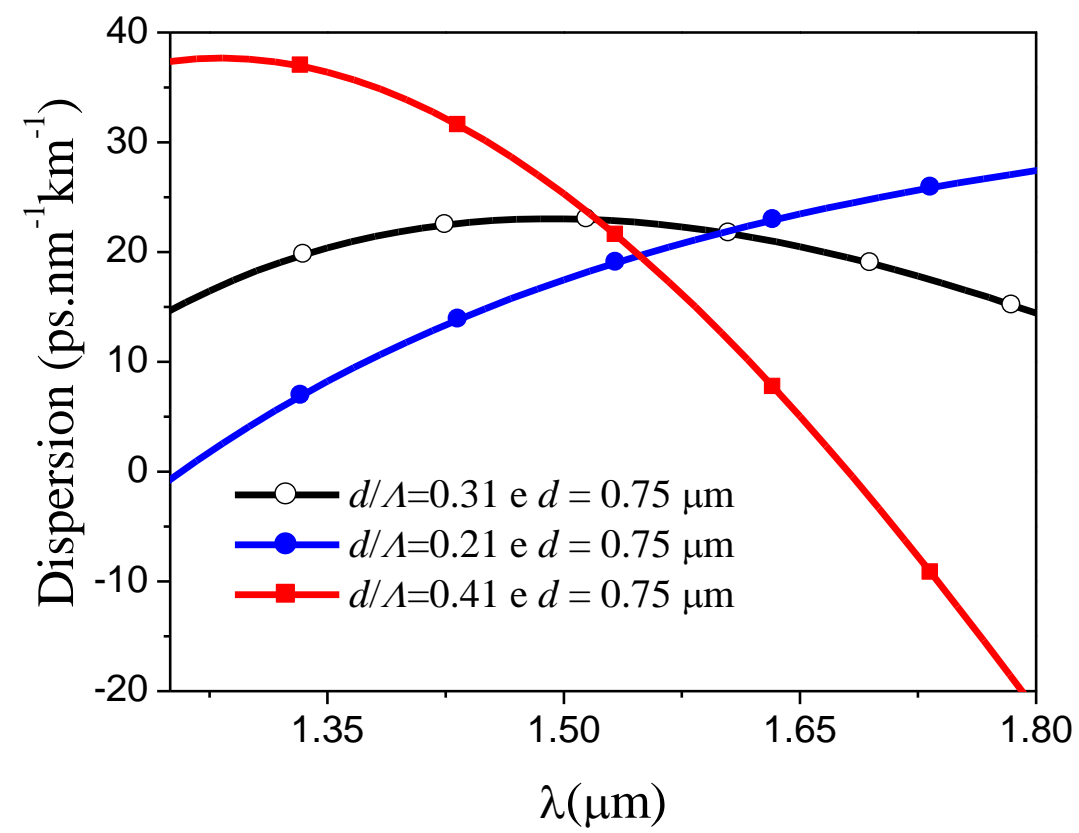

Fig. 7. Chromatic dispersion for the fundamental mode of the structure with twelve-fold symmetry considering variations of $d / \Lambda$ for a range of wavelength varying from $1.25 \mu \mathrm{m}$ to $1.8 \mu \mathrm{m}$.

It is observed that small variations in the ratio $d / \Lambda$ implies large changes in the dispersion curve and consequent displacement of the point of maximum dispersion, producing non-flat dispersion values within the corresponding optical spectrum bands $E, S, C$ and $L$.

In all simulations the thickness of the cylindrical PML was $1 \mu \mathrm{m}$, i.e., $d_{P M L}=1.0 \mu \mathrm{m}$, with this thickness, the used computational window was free of any possibility of undesirable reflections within the established limits. It's important to note that to obtain a wider range of wavelengths with ultra flat chromatic dispersion, the optimization of the structure shown in Fig. 1 must be obtained and will be used the evolutionary techniques such as Genetic Algorithms. One possible application of this fiber model as directional coupler is under review. The Fig. 8 shows the modal field distribution for component $h_{x}$ of PQCF. This result shows through the field distribution, the energy stored in the central core of the structure, which has as characteristic confine the fundamental mode in the region with no air holes, also known as the defective region or a region with higher quantity of core solid. 


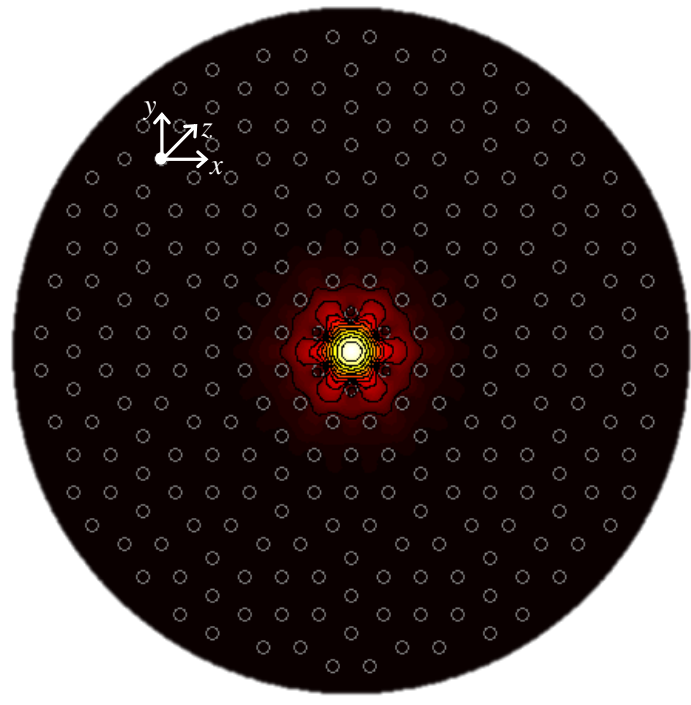

(a)

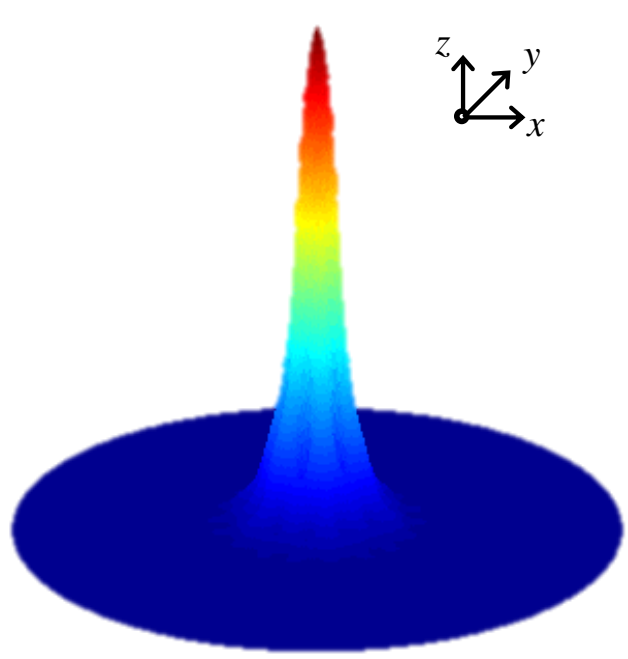

(b)

Fig. 8. Field distribution for the fundamental mode with $\lambda=1.55 \mu \mathrm{m}$ (a) Level Curves and (b) Surface.

In the next application, the structure shown in Fig. 1 was considered. Here, the PQCF with 12-fold symmetry considering $d=0.75, d / \Lambda=0.31$ and $\lambda=1.55 \mu \mathrm{m}$, was applied to obtain the coupling distance for PQCF with three cores. The structure was stimulated with a beam launched in the central core and the maximum power transfer occurred for a distance around $2.750 \mu \mathrm{m}$.

In the analysis of the directional coupler using the PQCF proposed in this work the coupling length $L$ obtained using the VBPM is in agreement with the value from the relation $L_{B}=\pi /\left(\beta_{\text {eff } 1}-\beta_{\text {eff } 2}\right)$ obtained through the modal analysis [4], where $L_{\mathrm{B}}$ is the beating length, and $\beta_{\text {eff1 }}$ and $\beta_{\text {eff2 }}$ corresponding the propagation constant of the super modes symmetrical and anti-symmetrical of lower order. This procedure is according with the coupled mode theory that consider the propagation modes of the fiber and the propagation distance $L_{\mathrm{B}}$, in this case the coupling coefficient is obtained according of PQCF characteristics considering the connections between cores of the fibers [20]. The comparison with the results obtained from modal analysis to confirm the accuracy of the results presented, since that modal analysis technique is recognized accurate for these types of application.

The Fig. 9 shows the normalized power existent in the cores 2-3 of the PQCF along the propagation direction. The black curve with the line without circles corresponds to the power variation of the central core 1 , the red curve with empty circles corresponds to the power coupled to the horizontal core 2 and the blue curve with full circles corresponds to the power coupled to horizontal core 3 . It may be noticed from the numerical results that the powers in the cores 2-3 gradually increase, while the whole power launched in the core 1 is entirely transferred to the adjacent cores in propagation distance of $2.750 \mu \mathrm{m}$. 


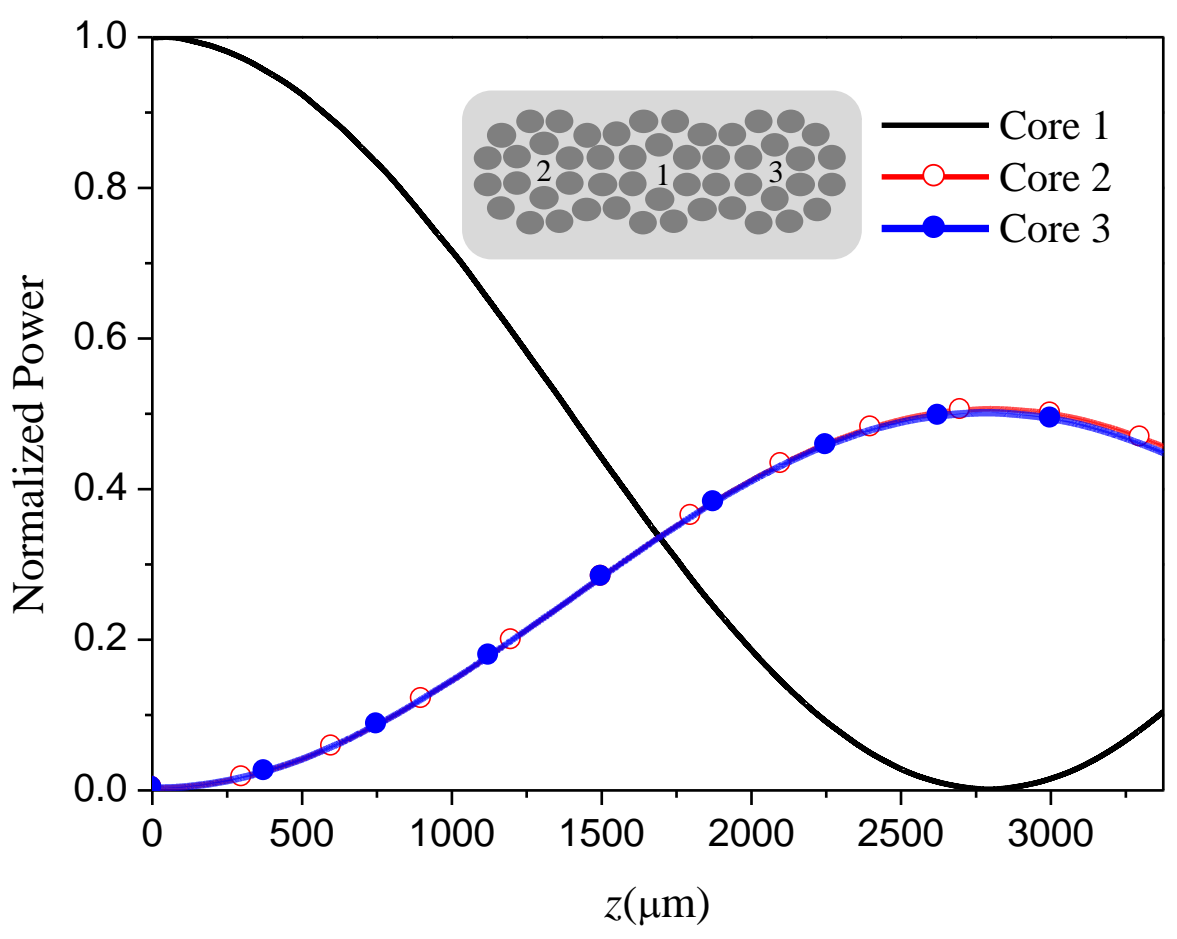

Fig. 9. Maximum distance of power transfer for PQCF 12-fold symmetry with three cores.

\section{CONCLUSIONS}

In this work the finite element method in conjunction with modal analysis, beam propagation method (BPM) and perfectly matched layers of cylindrical type were successfully applied to the analysis of a PQCF 12-fold symmetry and its application as photonic coupler. The parsed structure exhibits an ultra-flat chromatic dispersion for a range of wavelengths varying from 1.4 to $1.6 \mu \mathrm{m}$ covering bands $E, S, C$ e $L$, with chromatic dispersion, for the silica doped with germanium, varying between 22.1 e $23.01\left[\mathrm{ps} \cdot \mathrm{km}^{-1} . \mathrm{nm}^{-1}\right]$. The results for the dispersion were obtained using a numerically efficient formulation based on the finite element method which includes dispersing of material.

Due to the relative small variation of approximately $4 \%$, this model of structure can be applied for compensation of chromatic dispersion within the range where it is ultra flat. The main contribution of this work is to obtain photonic quasicrystal fiber with 12 -fold symmetry and the core composed by silica doped with germanium. Preliminary results show that the percentage of doping can influence the variation of the chromatic dispersion as a function of wavelength.

In addition when the structure composed by three cores is considered, the power launched in central core has been equally divided between the two neighbor and equidistant cores with a coupling coefficient of 50\%. It has been noticed that as the guiding mechanism is the total internal reflection, the confinement losses can be neglected in the structures analyzed in this work. 


\section{ACKNOWLEDGMENT}

The authors would like to thank the Federal University of Rio Grande do Norte (UFRN), the Federal University of Semiarid Region (UFERSA) and the Financing Agency of Studies and Projects (FINEP) for the physics support, and INCT Fotonicom and CNPq for financial support.

\section{REFERENCES}

[1] A. C. S. Júnior, "Recent progress and novel applications of photonic crystal fibers", Report on Progress in Physics, v. 73, pp. 1-21, 2010.

[2] S. Kim, C. S. Kee and J. Lee, "Novel Optical Properties of Six-Fold Symmetric Photonic Quasicrystal Fibers", Optics Express, v. 15, pp. 13221-13226, 2007.

[3] S. Kim and C. S. Kee, "Dispersion Properties of Dual-Core Photonic Quasicrystal Fibers", Optics Express, v. 15, pp. 15885-15890, 2009.

[4] L. Yu-He, F. Wan-De and S. Qiu-Qin, "A novel Photonic Quasicrystal Fiber with Broadband Large Negative Dispersion”, Chine Physics Letters, v. 27, pp. 114211-1-4, 2010.

[5] A. Méndez e T. F. Morse, Specialty Optical Fibers Handbook, San Diego, Academic Press, 2007

[6] J. P. da Silva, "Ge-Doped Microstructured Fiber Design by Genetic Algorithm for Directional Coupling”, Microwave and Optical Technology Letters, v. 55, pp. 281-285, 2013.

[7] Paulo Sérgio Patrício e Marcos A. R. Franco, "Fibras Ópticas Micro-estruturadas para Compensação de Dispersão Cromática”, 14 SBMO - Simpósio Brasileiro de Microondas e Optoeletrônica e $9^{\circ}$ CBMag - Congresso Brasileiro de Eletromagnetismo- MOMAG 2010, pp 615-620, 2010.

[8] D. B. Mortimore, “Wavelength-flattened fused couplers,” Electron. Lett., v. 21, pp. 742-743, Aug. 1985.

[9] D. B. Mortimore, "Theory and fabrication of $4 \times 4$ single-mode fused optical fiber couplers," Appl. Opt., v. 29, pp. 371374, Jan. 1990.

[10] A. Kumar, R. K. varshney, and R. K. Sinha, "Scalar modes and coupling characteristics of eight-port waveguide couplers,” J. Light. Technol., v. 7, pp. 293-296, Feb. 1989.

[11] H. E. Hernández- Figueroa, F. A Fernández, Y. Lu and J. B. Davies, "Vectorial finite element modelling of 2D leaky waveguides”, IEEE Trans. Magn. v. 31, pp. 1710-1712, 1995.

[12] J. P. da Silva, H. E. Hernández-Figueroa and A. M. F. Frasson, "Improved Vectorial finite-element BPM analysis for transverse anisotropic media”, J. Lightwave Technol., V. 21, pp. 567-576, 2003.

[13] J. P. da Silva, D. S. Bezerra and V. F. Rodriguez-Esquerre, I. E. da Fonseca, "Ge-Doped Defect-Core Microstructured Fiber Designby Genetic Algorithm for Residual Dispersion Compensation”, IEEE Phot, Techn. Letters, v. 22, pp. 1337 1339, 2010.

[14] J. Buck, Fundamentals of Optical Fibers. Hoboken, NJ: Wiley-Interscience, 1995.

[15] M. A. R. Franco, V. A. Serrão, and F. Sircilli, "Microstructured Optical Fiber for Residual Dispersion Compensation Over S + C + L + U wavelength Bands", IEEE Photonics Technology Letters, v. 20, pp. 751-753, 2008.

[16] J. P. da Silva and E. R. M. Dantas, "Modal Analysis of a Photonic Quasicrystal Fiber Doped with Germanium" Microwave \& Optoelectronics Conference (IMOC), 2013 SBMO/IEEE MTT-S International, IEEE, 2013.

[17] J. P. da Silva, D. S. Bezerra and H. E. Hernàndez-Figueroa, "Step index holey fiber design by genetic algorithm for chromatic dispersion compensation”, Microwave and Optical Technology Letters, v. 53, pp 1585-1588, 2011.

[18] S. Lee, W. Ha, J. Park, S. Kim and K. Oh, "A new design of low-loss and ultra-flat zero dispersion photonic crystal fiber using hollow ring defect, Optics Communications, v. 285, pp 4082-4087, 2012.

[19] K. Saitoh, N. Florous and M. Koshiba, "Ultra-flattened chromatic dispersion controllability using a defected-core photonic crystal fiber withlow confinement losses,” Opt. Express, V. 13, pp. 8365-8371, 2005. 

http://dx.doi.org/10.1590/2179-10742015v14i2481

[20] S. K. Varshney, K. Saitoh, R. K. Sinha and M. Koshiba. "Coupling Characteristics of Multicore Photonic Crystal FiberBased 1x4 Power Splitters”. Journal of Lightwave Technology, V. 27, pp 2062-2068, 2009. 